\title{
The Effect of Performance Experience on Vocal Music Majors' Perception of Musical Tension
}

\author{
Jeremy S. Lane \\ School of Music \\ University of South Carolina
}

\begin{abstract}
Participants $(N=20)$ enrolled in two separate applied studios listened to the same art song during their weekly lesson for six consecutive weeks, using a CRDI dial to measure perceived musical tension as they listened. In addition, members of one studio $(n=10)$ studied and prepared the specific song used during listening tasks. Similar to methods used in previous research in this area, the investigator did not provide a definition of musical tension; all participants were allowed to define this construct in their own manner. Results indicated a high degree of consistency among responses, both in comparisons of overall means of participant groups and in comparisons of week-by-week means within each group. Participants in this study generally perceived tension in a similar manner, and did not seem to demonstrate any significant changes in perception of tension due to performance experience, increased musical knowledge, or repeated hearings of the song.
\end{abstract}

Keywords: music perception, musical tension; CRDI; music performance 
A commonly held belief among many music educators is the need for comprehensive knowledge to facilitate meaningful performance. Most would agree that performing a piece of music successfully is more than simply playing or singing correct notes and rhythms at the correct volume. As noted scholar David Elliott (1995) writes, "A performance projects a musician's understanding of the several dimensions of a given composition into a definite context... [S] $]$ uccess in musical performing depends on understanding all relevant dimensions of a given composition" (p. 165).

Findings of research in the music education literature suggest that musical performance can go beyond synthesis and projection of assimilated knowledge, but can also increase and develop deeper musical understanding for the performer. Kjellend and Kerchner (1998) conducted a review of research related to music performance training and its affects on subsequent listening experience. Findings among these studies suggest that music performance training can be, among other things, beneficial for perception and refinement of cognitive processing skills. The authors noted, however, a lack of research investigating the influence of performance experience on affective response and suggested that continued research should "...explore the combination of both perceptual and affective dimensions of experiences in music" (p. 48).

A key obstacle confronting researchers who wish to investigate the nature of affective response is the measurement of responses in real time while the participant is engaged with the artwork. In an effort to deal with this issue, researchers at Florida State University and the University of Missouri-Kansas City developed the Continual Response Digital Interface (CRDI). The CRDI is a dial that the responder moves back and forth in relation to their perceptions of specific elements (or combinations thereof) in music. The dial has been used successfully in a variety of settings and has been shown to be a reliable tool for measuring affective response (Geringer, Madsen, \& Gregory, 2004).

In recent years, researchers have studied perception of musical tension as a unique component of overall aesthetic experience. Research has demonstrated that musical tension tends to be perceived differently and with wider variations in magnitude than does aesthetic response and seems to be a construct more easily relatable to non-musicians (Fredrickson, 1995; Fredrickson \& Coggiola, 2003; Lynchner, 1998).

Analyses of perception of musical tension suggest that it is a construct that tends to be perceived similarly across varying participant groups. Researchers have compared groups of musicians to non-musicians (Fredrickson \& Coggiola, 2003; Fredrickson, 1999, 2000, 2001; Madsen \& Fredrickson, 1993), elementary, middle, and high school students (Fredrickson, 1997). A common theme among these studies is that participants perceive occurrences of high and low tension similarly, though there may be differences in the magnitude of their perception. Other studies have shown that perception of tension does not seem to be influenced by performance medium (Fredrickson, 2001), translation of text in vocal music (Hackworth \& Fredrickson, 2010), or performer's use of rubato (Fredrickson 
\& Johnson, 1996). These findings are particularly noteworthy when one considers that in all of the studies cited above, the term 'musical tension' was not assigned a specific definition; the participants were left to their own devices to define 'tension' in their own manner.

To date, there are few published studies that have investigated the effect of performance experience on perceived musical tension within a specific piece of music. Fredrickson (1999) studied the relationship between performance experience and perception of tension in a selected piece of band music, Holst's First Suite in Eb, among university band students, university choral students, and high school band students. Results indicated minimal differences in perception of tension between participants with experience performing the piece than participants with no performance experience with the work.

The purpose of this study was to continue the line of research investigating the role of performance experience on perception of musical tension. Given the unavoidable repeated hearings inherent in the process of preparing music for performance, a secondary purpose was to examine any changes in perception of tension across multiple hearings of the same piece. The specific research questions that guided this study were:

1. Can perception of musical tension change in relation to increased performance knowledge and experience?

2. Can perception of musical tension change in relation to multiple repeated hearings of the same piece of music?

\section{METHODOLOGY}

Participants in this study were undergraduate vocal music majors $(N=20)$ enrolled in two separate applied studios at a large Southeastern university ${ }^{1}$. Members of Studio A $(n=10)$ served as a treatment group, and members of Studio B $(n=10)$ served as a control group.

The investigator conducted this research in two phases. In Phase 1, members of Studio A listened to the same art song during their weekly lesson for three consecutive weeks. As they listened, they manipulated the CRDI dial in relation to how they perceived musical tension. Similar to methods used in previous research in this area, the investigator did not provide a definition of musical tension; all participants were allowed to define this construct in their own manner.

Prior to the first week of listening, the studio teacher read aloud the following directions to each participant:

\footnotetext{
1 The investigator wishes to thank the two studio teachers for sharing their valuable lesson time in data collection for this project. Both were extremely gracious and helpful, and the investigator is greatly indebted to them for all their efforts.
} 
You are about to listen to a single art song by Franz Schubert. This study is an attempt to provide ongoing information about perception of tension in music. You will be using the dial in front of you to record your responses to the music. As you listen, move the dial along the curve in relationship to what you consider to be various degrees of musical tension. There are no right or wrong answers. Please begin with the dial on the far left of the curve. When the music starts, move the dial as you hear the tension increase or decrease in the music. You may move the dial as far and as often as you want. If you have any questions, please ask them now.

The directions given to participants in this study were similar to those used in prior research (Fredrickson, 1999). The directions were only read during the first week and were not repeated during the $2^{\text {nd }}$ and $3^{\text {rd }}$ weeks of listening.

In Phase 2, Studio A participants completed a second 3-week sequence of listening/responding (identical in procedure to Phase 1); in addition, Studio A participants studied the song as part of their studio repertoire and prepared the piece for performance on their end-of-semester juries. To supplement the learning process, the applied teacher for Studio A provided each of their students with a packet containing background information about the piece, the text of the song with complete translation, phonetic marks used for diction, and historical information related to the music.

Also during Phase 2, Studio B participants completed a procedure of listening and responding with the CRDI dial identical to that used with Studio A in Phase 1. Studio B participants did not learn or perform the song, nor did they receive any additional information about the music; they only listened and responded using the CRDI dial. Studio B participants received the exact same instructions as did participants in Studio A, and were allowed to define musical tension in their own manner.

The music used for all phases and activities in this study was a single German lied by Franz Schubert titled 'Nur wer die Sehnsucht Kennt' (D. 481, 3rd setting, Naxos Catalogue No. 0021852BC) performed by Arleen Auger, soprano, and Walter Olbertz, piano. During the three-week period in which Studio A sang and worked on the piece, the applied instructor provided a version of the song that was appropriate for their particular voice range. In some cases, this resulted in the song being transposed to another key. All participants in both studios listened to the same song in the same key, even though some participants in Studio A may have sung the song in a key differing from the one they listened to while using the CRDI dial.

The range of the CRDI dial used by participants went from 0 (perception of no musical tension) to 256 (perception of maximum musical tension). The investigator calibrated the dial to record its position at half-second intervals. The total duration of the song was 211 seconds ( $3^{\prime} 31$ "), which resulted in 422 recordings of the dial position within each individual file. 
Phase 1 of data collection yielded 3 files for each member of Studio A (one per week for 3 weeks), and produced a total of 30 data files for analysis. The procedure for Phase 2 resulted in 60 additional CRDI data files (30 from Studio A and 30 for Studio B). Using this data, the investigator calculated group means for each week within both phases, and also calculated overall group means for each phase (Studio A Phase 1, Studio A Phase 2, Studio B Phase 2). The investigator used these means to 1) produce graphs that provided visual representation of data, and 2) run Pearson Product-Moment correlation tests to compare the shapes of graphs among specific groups.

\section{RESULTS}

\section{Phase 1}

Table 1 shows the results of Person Product-Moment correlations among each week of Studio A for Phase 1. Results indicate a strong consistency of responses across all weeks of Phase 1. Correlation coefficients indicate that perceptions of tension remained virtually unchanged across the 3-week test period, with all $r$-values over 0.90 for each comparison.

\section{Table 1}

Pearson r-values for correlations among each week of Phase 1 for Studio A.

\begin{tabular}{llll}
\hline Studio A & Week 1 & Week 2 & Week 3 \\
\hline Week 1 & -- & $0.951^{*}$ & $0.946^{*}$ \\
Week 2 & $0.951^{*}$ & -- & $0.948^{*}$ \\
Week 3 & $0.946^{*}$ & $0.948^{*}$ & -- \\
\hline
\end{tabular}

* Correlation is significant at the 0.01 level (2-tailed).

Figure 1 presents a graph illustrating the curves for each week of Studio A, Phase 1. Included on the graph are approximate markings of the musical form of the song, as well as corresponding time frames for each section. In general, participants perceived a gradual increase in tension through the first two minutes of the song (with a couple of instances of rising and falling of perceived tension within this time period), with peaks of perceived tension occurring in the middle of the B section and beginning of the second A section before a gradual decline through the end. 


\section{Journal of Research in Music Performance}

Winter 2012

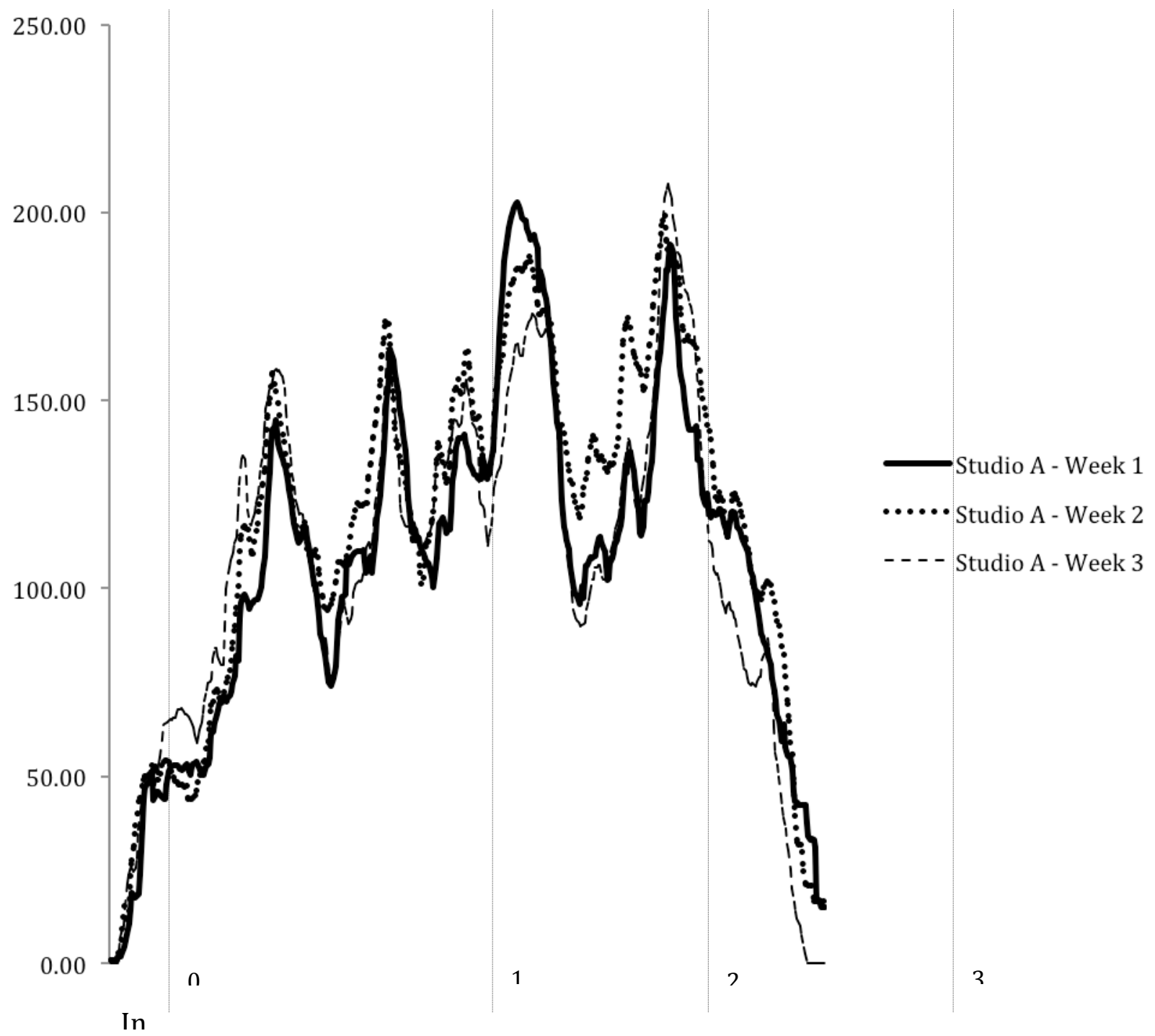

Figure 1: CRDI Dial Graphs for Studio A, Phase 1

\section{Phase 2}

Table 2 shows the results of Pearson-Product Moment correlations for each week of Studio A Phase 2. Results indicate very strong correlations across weeks 4-6, with all coefficients exceeding 0.970 in each comparison.

\section{Table 2}

Pearson r-values for correlations among each week of Phase 2 for Studio A.

\begin{tabular}{clll}
\hline Studio A & Week 4 & Week 5 & Week 6 \\
\hline Week 4 & -- & $0.978^{*}$ & $0.974^{*}$ \\
Week 5 & $0.978^{*}$ & -- & $0.984^{*}$ \\
Week 6 & $0.974^{*}$ & $0.984^{*}$ & -- \\
\hline
\end{tabular}

* Correlation is significant at the 0.01 level (2-tailed). 
Figure 2 presents a graph illustrating the curves for each week of Phase 2 for Studio A. Visual analysis of the graph indicates that the overall pattern observed during Phase 1 (gradual increase in perceived tension during first two minutes, peaks of tension in middle of $B$ and beginning of second A sections, decline through the end) remained consistent during Phase 2.

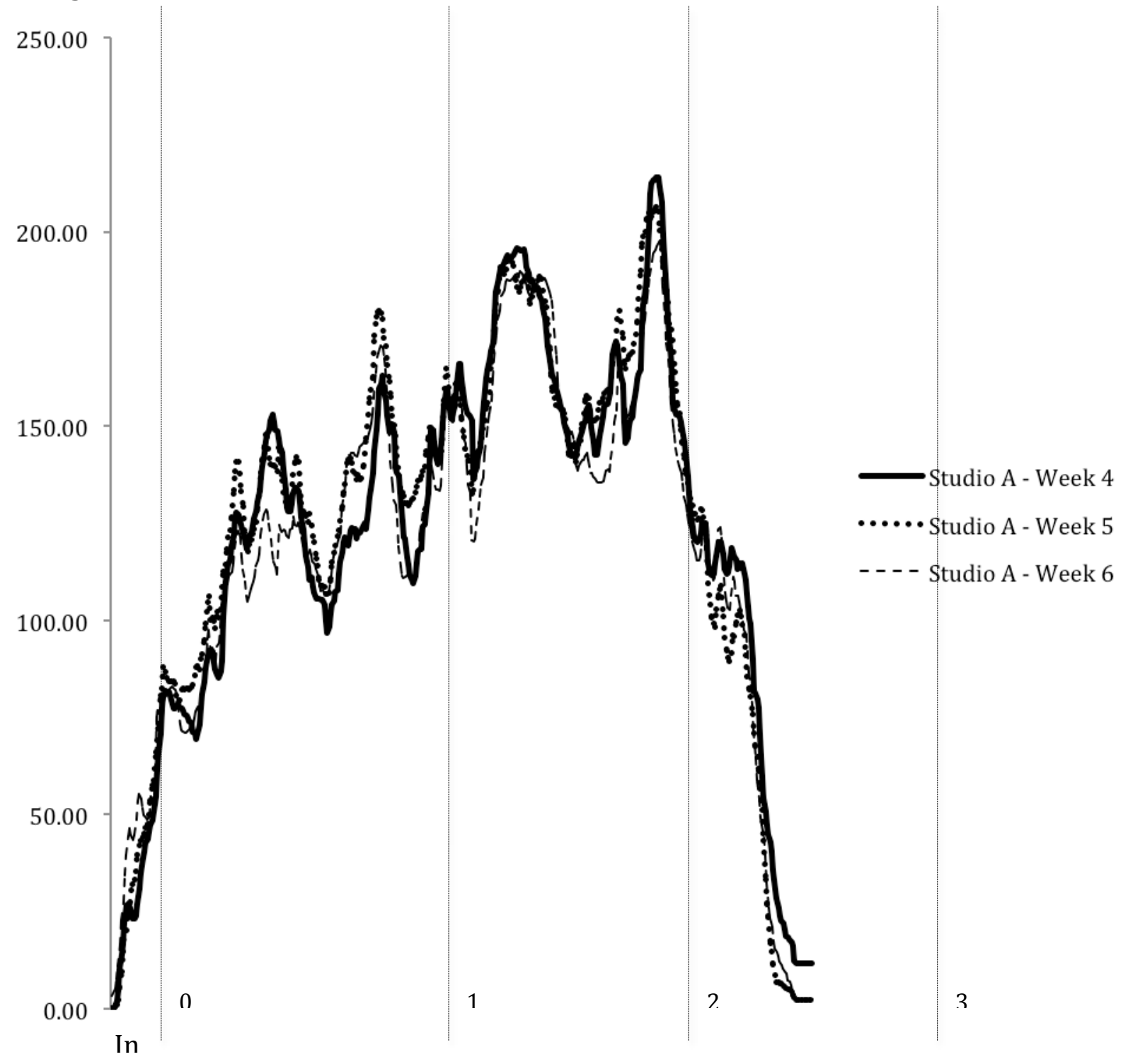

Figure 2: CRDI Dial Graphs for Studio A, Phase 2

Table 3 lists the $r$-values for correlations across the entire 6-week experimental period (both Phases 1 and 2) for Studio A. Results indicate that perception of tension did not seem to change markedly across the six-week period. There is a slight gradual decrease in consistency of responses (as noted by decreasing $r$-values week by week through Week 5), though the overall correlations among all weeks are statistically significant with $r$ values of 0.898 or higher for each comparison. 
Table 3.

Pearson r-values for correlations of Studio A Phases 1 and 2.

\begin{tabular}{llll|lll}
\hline & Week 1a $^{\text {a }}$ & Week 2 & Week 3 & Week 4 & Week 5 & Week 6 \\
\hline Week 1 & -- & $0.951^{*}$ & $0.946^{*}$ & $0.938^{*}$ & $0.898^{*}$ & $0.901^{*}$ \\
Week 2 & $0.951^{*}$ & -- & $0.948^{*}$ & $0.952^{*}$ & $0.917^{*}$ & $0.917^{*}$ \\
Week 3 & $0.946^{*}$ & $0.948^{*}$ & -- & $0.949^{*}$ & $0.932^{*}$ & $0.918^{*}$ \\
Week 4 & $0.938^{*}$ & $0.952^{*}$ & $0.949^{*}$ & - & $0.978^{*}$ & $0.974^{*}$ \\
Week 5 & $0.898^{*}$ & $0.917^{*}$ & $0.932^{*}$ & $0.978^{*}$ & -- & $0.984^{*}$ \\
Week 6 & $0.901^{*}$ & $0.917^{*}$ & $0.918^{*}$ & $0.974^{*}$ & $0.984^{*}$ & -- \\
\hline
\end{tabular}

* Correlation is significant at the 0.01 level (2-tailed).

a Weeks 1-3 = Phase 1; Weeks 4-6 = Phase 2

Table 4 shows the results of Pearson-Product Moment correlations for each week of Studio B Phase 2. Correlations indicate consistent patterns of responses across the three weeks for Studio B. Analysis reveals that a similar pattern of response across time observed during both phases for Studio A is also reflected in responses for Studio B. Patterns of responses for Studio B reflect a high degree of consistency, with $r$-values exceeding 0.899 in each case.

\section{Table 4}

Pearson r-values for correlations of Studio B Phase 2.

\begin{tabular}{clll}
\hline Studio B & Week 4 & Week 5 & Week 6 \\
\hline Week 4 & -- & $0.899^{*}$ & $0.926^{*}$ \\
Week 5 & $0.899^{*}$ & -- & $0.945^{*}$ \\
Week 6 & $0.926^{*}$ & $0.945^{*}$ & -- \\
\hline
\end{tabular}

${ }^{*}$ Correlation is significant at the 0.01 level (2-tailed).

Figure 3 presents a graph illustrating the curves for each week of Phase 2 for Studio B. There appears to be noticeable differences in the peaks of perception, specifically in middle of section B (1:50) and at the beginning of the second A section (2:20). These differences did not seem to affect significantly the comparisons of week-by-week means, as all correlations across the three-week period were $r=0.899$ or higher and were all statistically significant. 


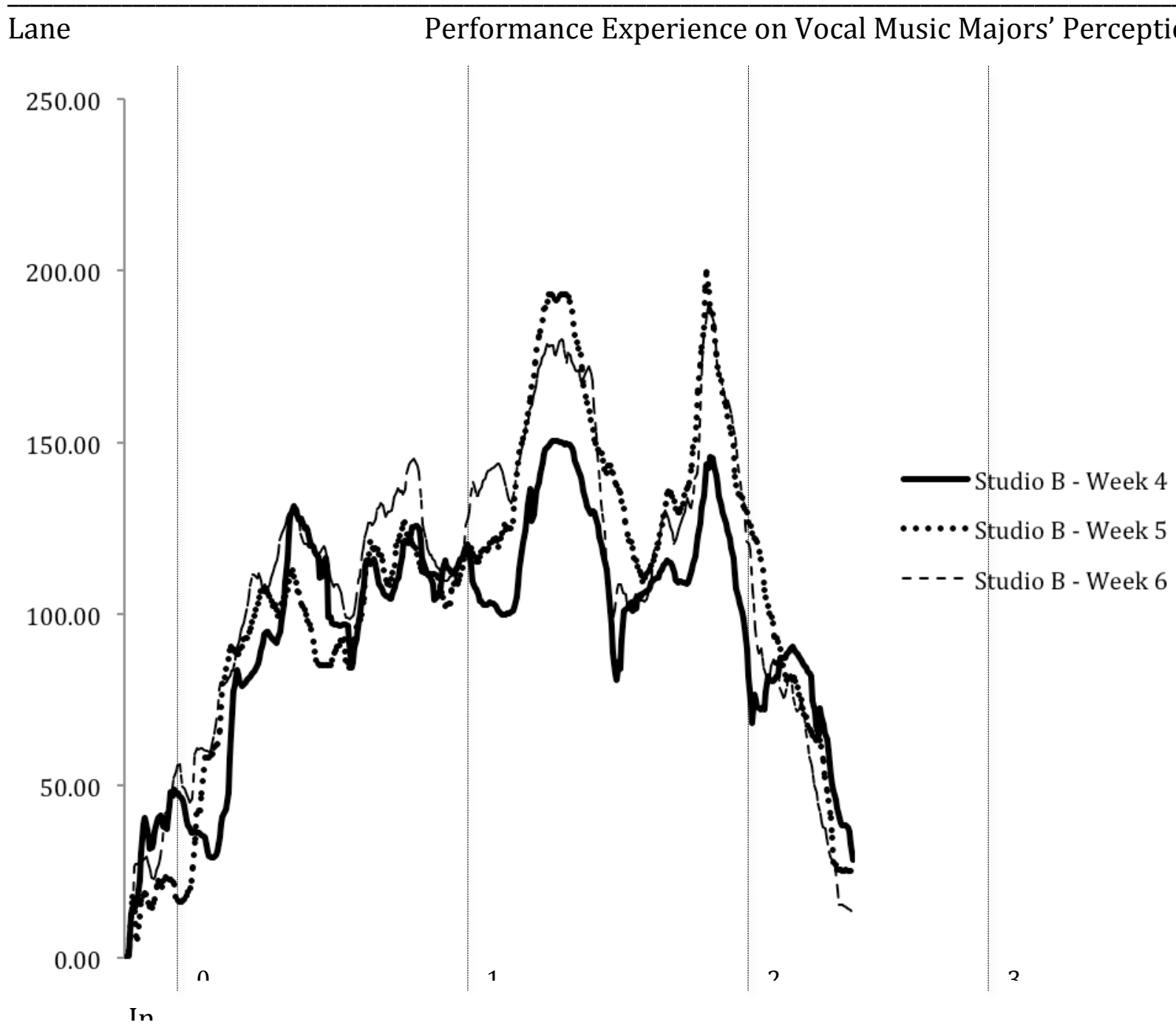

Figure 3: CRDI Dial Graphs for Studio B, Phase 2

In order to compare groups, the investigator calculated overall means and generated graphs for each participant group within each 3-week period (Studio A Phase 1, Studio A Phase 2, Studio B Phase). The investigator used these overall group means for Pearson Product-Moment correlation tests. Table 5 lists the correlation coefficients for comparisons of overall group means among Studio A Phase 1, Studio A Phase 2, and Studio B Phase 2.

\section{Table 5}

Pearson r-values for correlations among Studio A Phase 1, Studio A Phase 2, and Studio B Phase 2.

\begin{tabular}{llll}
\hline & Studio A Phase 1 & Studio A Phase 2 & Studio B Phase 2 \\
\hline Studio A Phase 1 & -- & $0.939^{*}$ & $0.953^{*}$ \\
Studio A Phase 2 & $0.939^{*}$ & -- & $0.943^{*}$ \\
Studio B Phase 2 & $0.953^{*}$ & $0.943^{*}$ & -- \\
\hline
\end{tabular}

* Correlation is significant at the 0.01 level (2-tailed). 
Results of correlation tests indicate a strong degree of consistency of responses among the three test groups; in each comparison, the obtained $r$-value exceeded 0.930 . Coefficients also indicate little difference in responses between Studio A Phase 1 and Studio A Phase 2, indicating that these participants perceived tension in a similar manner and with little change across the entire 6-week testing period.

Figure 4 displays the curves representing the overall mean response for each group. The consistency among group means is reflected in the overall shapes of each curve and indicate little evidence of changes in perception that may be due to any resultant effect from performance experience or repeated hearings of the specific piece. This is especially noteworthy when comparing the two curves from Studio A Phase one to Studio A Phase 2, in which the curves are essentially identical.

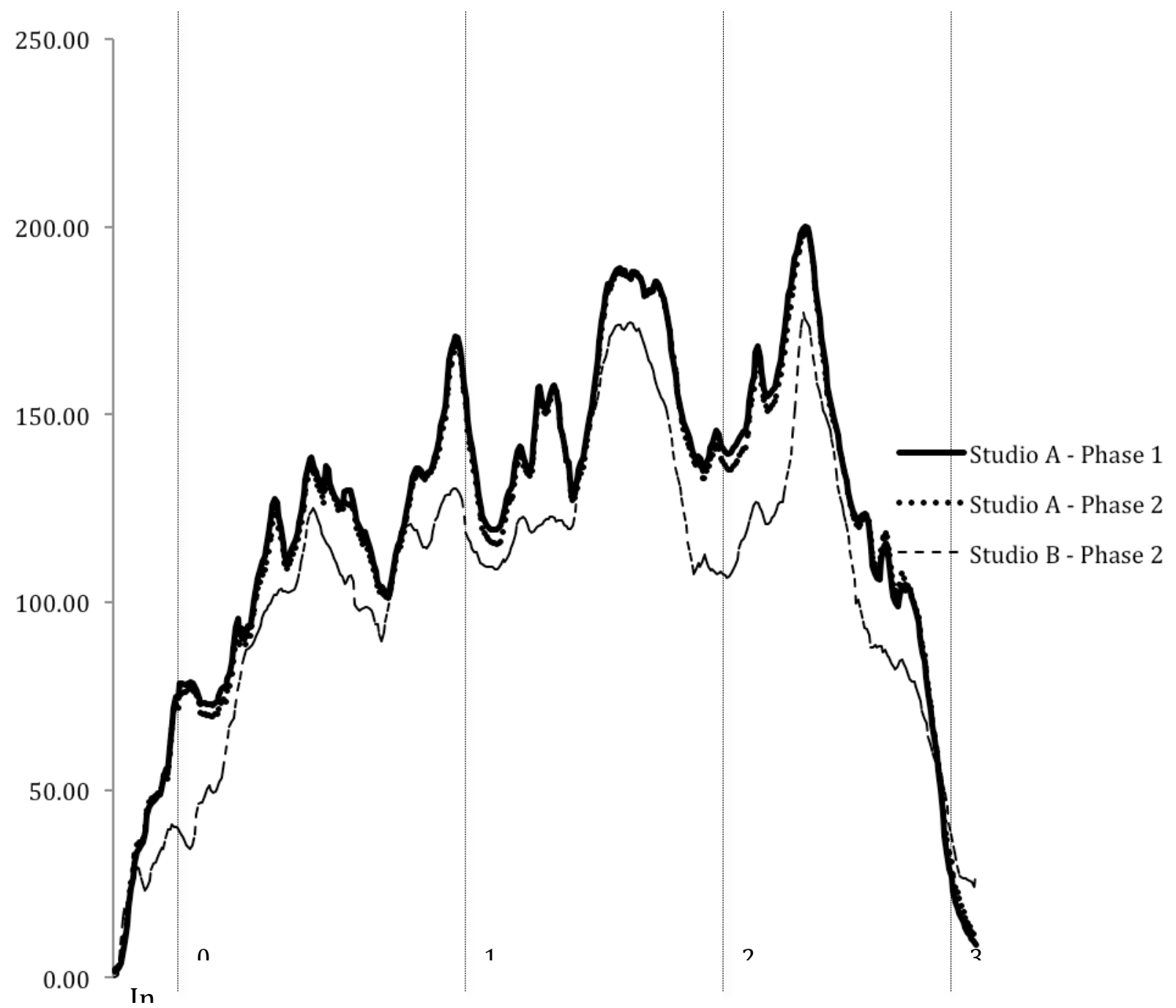

Figure 4: CRDI Dial Graphs for Studio A, Phase 1 and 2, and Studio B, Phase 2. 
Lane

Performance Experience on Vocal Music Majors' Perception

\section{DISCUSSION}

There are certain limitations in the design of this study that need to be considered prior to interpretation of results. The use of intact studios limited the available participants to a number noticeably smaller than what might be ideal for this type of project. In general, other studies in this line of research have compared larger samples, usually in the range of 50-100 total participants. In addition, the use of weekly lesson time for activities related to this study forced the issue of brevity. To avoid burdening the applied studio teachers with activities not primarily related to their specific plans for each student that semester, the investigator specifically chose data collection methods that would (ideally) take less than five minutes of time each lesson. Given these two limitations (participant pool size; short durations of activities), it would not be prudent to generalize the results of this study to broader populations.

The investigator undertook this project in an effort to provide information on the relationship between performance experience and the perception of musical tension. Results suggest a remarkable consistency of responses, both in comparisons of overall means of participant groups and in comparisons of week-by-week means within each group. When listening to the selected piece, participants in this study generally perceived tension in a similar manner, and did not seem to demonstrate any significant changes in perception of tension due to performance experience, increased musical knowledge, or repeated hearings of the song.

Overall, the findings of this study support similar themes reported in the extant research literature. Results of numerous studies suggest that perception of musical tension is a fairly stable across a wide array of participant groups, and is not significantly altered by external influences such as performance medium, performance experience, or other variables related to musical performance such as rubato. One finding that was unique to this study was the slight decline in consistency of responses among Studio A participants across the six-week testing period. The data does not provide enough evidence or perspective to make meaningful inferences as to why this may have occurred; it may be a simple variation in numbers, or it may point towards a more meaningful trend. Future research may help clarify the implications of this finding

An important issue that deserves deeper investigation is related to the timing of the measurement procedures (i.e. continuous or post hoc measurement of experiences). In an investigation of perception of musical intensity, Duke \& Colprit (2001) observed that participant's 'in-the-moment' (or continuous) responses were significantly different than responses given at the conclusion of the musical excerpt. These findings led the authors to conclude "...momentary responses are often different than subjects' post hoc perceptions of temporal experiences” (pg. 340). In a similar manner, it may be that 'in-performance' responses are often different than post hoc or continuous perceptions of temporal experiences. 
Given that, at least at this time, continuous measurement of affective response during performance is prohibitively difficult the differences between continuous and post hoc measures may be a factor in the results of this study. It may be that performers do have a deeper affective connection to the music when engaged in performing as opposed to listening and responding removed from performance activities. It seems that the next step in the research process in this area should focus on identifying successful data collection tools/techniques for use in measuring affective responses during the act of performance. By providing methods for 'in-performance' measurements, investigations may proceed in manners that provide a clearer perspective on the influence of performance on musician's affective response.

\section{REFERENCES}

Duke, R. A., \& Colprit, E. J. (2001). Summarizing listener perceptions over time. Journal of Research in Music Education, 49, 330-342.

Elliott, D. A. (1995). Music matters. New York: Oxford University Press.

Fredrickson, W. E. (1995). A comparison of perceived musical tension and aesthetic response. Psychology of Music, 23, 81-87.

Fredrickson, W. E. (1997). Elementary, middle, and high school students' perception of tension in music. Journal of Research in Music Education, 45, 626-635.

Fredrickson, W. E. (1999). The effect of musical performance on perception of tension in Gustav Holst's First Suite in Eb. Journal of Research in Music Education, 47, 44-52.

Fredrickson, W. E. (2000). Perception of tension in music: Musicians versus non-musicians. Journal of Music Therapy, 37, 40-50.

Fredrickson, W. E. (2001). The effect of performance medium on perception of musical tension. Bulletin of the Council for Research in Music Education, 47, 44-52.

Fredrickson, W. E. \& Coggiola, J. C. (2003). A comparison of music majors' and nonmajors' perceptions of tension for two selections of jazz music. Journal of Research in Music Education, 51, 259-270.

Fredrickson, W. E. \& Johnson, C. M. (1996). The effect of performer rubato on listener perception of tension in Mozart. Psychomusicology, 15, 78-86.

Geringer, J. M., Madsen, C. K., \& Gregory, D. (2004). A fifteen-year history of the Continuous Response Digital Interface: Issues relating to validity and reliability. Bulletin of the Council for Research in Music Education, 160, 1-15. 
Hackworth, R. S. \& Fredrickson, W. E. (2010). The effect of text translation on perceived musical tension in Debussy's Noel des infants qui n'ont plus de maisons. Journal of Research in Music Education, 58, 184-195.

Kjelland, J. M., \& Kerchner, J. L. (Eds.). (1998). The effects of music performance participation on the music listening experience: A review of literature. Bulletin of the Council for Research in Music Education, 136, 1-55.

Lynchner, J. A. (1998). An empirical study concerning terminology relating to aesthetic response in music. Journal of Research in Music Education, 46, 303-319.

Madsen, C. K., \& Fredrickson, W. E. (1993). The experience of musical tension: A replication of Nielsen's research using the Continuous Response Digital Interface. Journal of Music Therapy, 30, 46-63. 